\title{
L'hétérogénéité des modes de cohabitation des 20-29 ans et des plus de 65 ans dans les régions françaises et espagnoles, à partir des données de recensement The heterogeneity of patterns of cohabitation of those aged between 20 and 29 and over 65 in French and Spanish regions, based on census data
}

\section{Béatrice Valdes}

Volume 41, numéro 2, automne 2012

La fin des recensements?

URI : https://id.erudit.org/iderudit/1013492ar

DOI : https://doi.org/10.7202/1013492ar

Aller au sommaire du numéro

\section{Éditeur(s)}

Association des démographes du Québec

ISSN

0380-1721 (imprimé)

1705-1495 (numérique)

Découvrir la revue

Citer cet article

Valdes, B. (2012). L'hétérogénéité des modes de cohabitation des 20-29 ans et des plus de 65 ans dans les régions françaises et espagnoles, à partir des données de recensement. Cahiers québécois de démographie, 41(2), 227-256. https://doi.org/10.7202/1013492ar

\section{Résumé de l'article}

Cette étude des modes de cohabitation des jeunes adultes âgés de 20 à 29 ans et des personnes âgées de plus de 65 ans, à partir des échantillons de recensement de la base IECM, permet de mettre en évidence une hétérogénéité spatiale des modes de cohabitation, avec non seulement une opposition entre la France et l'Espagne, mais aussi des disparités infranationales entre les régions françaises et espagnoles. On découvre une continuité au-delà de la frontière en matière de corésidence familiale des personnes âgées, résultant d'une permanence des modèles familiaux traditionnels dans le nord de l'Espagne et dans les régions du sud de la France. Les disparités concernant la fréquence de la cohabitation des jeunes adultes sont moins fortes, et correspondent à un phénomène nouveau, plutôt homogène et détaché des " modèles familiaux traditionnels ". La vision cantonale, obtenue à partir des données cantonales du recensement français de 2006, permet de mettre en lumière des différences infrarégionales importantes en matière de corésidence familiale. On discerne clairement une concentration particulière du modèle traditionnel de la « famille souche » dans l'extrême sud-ouest de l'Aquitaine.
Tous droits réservés @ Association des démographes du Québec, 2012
Ce document est protégé par la loi sur le droit d'auteur. L'utilisation des services d'Érudit (y compris la reproduction) est assujettie à sa politique d'utilisation que vous pouvez consulter en ligne.

https://apropos.erudit.org/fr/usagers/politique-dutilisation/ 


\title{
Cahiers québécois de démographie
}

Vol. 41, $\mathrm{n}^{\mathrm{o}}$ 2, automne 2012, p. 227-256

\section{L'hétérogénéité des modes de cohabitation des 20-29 ans et des plus de 65 ans dans les régions françaises et espagnoles, à partir des données de recensement}

\author{
BÉATRICE VALDES*
}

\begin{abstract}
Cette étude des modes de cohabitation des jeunes adultes âgés de 20 à 29 ans et des personnes âgées de plus de 65 ans, à partir des échantillons de recensement de la base IECM, permet de mettre en évidence une hétérogénéité spatiale des modes de cohabitation, avec non seulement une opposition entre la France et l'Espagne, mais aussi des disparités infranationales entre les régions françaises et espagnoles. On découvre une continuité au-delà de la frontière en matière de corésidence familiale des personnes âgées, résultant d'une permanence des modèles familiaux traditionnels dans le nord de l'Espagne et dans les régions du sud de la France. Les disparités concernant la fréquence de la cohabitation des jeunes adultes sont moins fortes, et correspondent à un phénomène nouveau, plutôt homogène et détaché des «modèles familiaux traditionnels». La vision cantonale, obtenue à partir des données cantonales du recensement français de 2006, permet de mettre en lumière des différences infrarégionales importantes en matière de corésidence familiale. On discerne clairement une concentration particulière du modèle traditionnel de la «famille souche » dans l'extrême sudouest de l'Aquitaine.
\end{abstract}

English abstract, p. 256

\section{INTRODUCTION}

\begin{abstract}
u vu des profondes mutations sociodémographiques qu'a connues Al'Europe depuis quelques décennies, certains auteurs ont évoqué une "seconde transition démographique en Europe" (Lesthaeghe, 1991; Lesthaeghe, 1995; Van de Kaa, 1987). Des bouleversements dans les comportements démographiques qui caractérisent l'entrée des individus dans la vie adulte ont eu lieu dans tous les pays d'Europe : retard de la
\end{abstract}

* Institut national d'études démographiques (INED) et Institut d'études démographiques de l'Université de Bordeaux (IEDUB), France (valdesbeatrice@hotmail.fr) 
décohabitation (Bozon et Villeneuve-Gokalp, 1995), recul de l'âge au mariage et augmentation de la dissolution de l'union (Desplanques et Saboulin, 1986; Haskey, 1993; Kiernan, 2002), hausse de la recomposition familiale et de la monoparentalité (Algava, 2002). La combinaison de différentes évolutions démographiques - notamment l'augmentation de l'espérance de vie (Bongaarts, 2006), la baisse de la fécondité et le recul du calendrier de la fécondité (Kohler, Billari et Ortega, 2002; Prioux, 2003; Sobotka, 2004) - a eu pour conséquence un vieillissement de la population. Or l'arrivée aux âges élevés des populations nées pendant le babyboom et l'amélioration de l'espérance de vie accélèrent ce vieillissement. Ces changements de comportements démographiques ont des conséquences sur la structure des ménages européens (Ogden et Hall, 2004) et posent la question des solidarités familiales.

Dans les pays européens, le vieillissement de la population pourrait avoir des conséquences directes sur les politiques publiques car il risque d'affecter la pérennité des financements des systèmes de sécurité sociale, et notamment la continuité des régimes de retraite (De Santis, 2003) qui permettent aux personnes âgées de vivre de manière indépendante.

De nombreuses études ont mis en exergue l'importance du rôle de la solidarité familiale - qui se traduit notamment par la corésidence entre générations successives d'adultes - lorsque surviennent des situations difficiles qui nécessitent un soutien (pauvreté, dépendance, rupture conjugale) ou lors de certaines phases clés du cycle de vie (transition vers l'âge adulte, veuvage) (Ayllon, 2009; Festy et Prokofieva, 2006; Holdsworth et Irazoqui Solda, 2002; Gaymu et collab. 2006).

La solidarité intergénérationnelle repose essentiellement sur les adultes âgés de 45 à 64 ans, qualifiés par certains de " génération pivot » car ils sont doublement sollicités par l'entraide familiale : ils doivent aider leurs enfants, jeunes adultes pas encore autonomes, et soutenir leurs parents âgés, souvent en début de dépendance (Attias-Donfut, 1995; Blöss, 2005; Fleury, Borsenberger, Cassilde et Lorentz, 2011). Jusqu'ici, le développement des systèmes de sécurité sociale au profit des plus vieux avait permis de libérer les membres de cette génération pivot du soutien financier aux personnes âgées. Mais ces systèmes de sécurité sociale risquent de ne plus être capables de subvenir aux besoins croissants d'une population vieillissante, avec une proportion de personnes dépendantes en recrudescence.

Dès lors, les questions des relations d'entraide familiale et de l'équilibre entre les générations, étant donné l'accroissement prévisible du nombre de personnes âgées, sont essentielles (Gaymu, Ekamper et Beets, 2007). De ce fait, de nombreuses études s'intéressent à la prise en charge 
par les solidarités familiales des besoins des personnes âgées en perte d'autonomie (Bonvalet et Ogg, 2009; Millar et Warman, 1996; Weber, 2007) et à la prise en charge de plus en plus tardive des jeunes adultes en voie d'autonomisation (Van de Velde, 2007; Galland, 2004). La solidarité familiale revêt une multitude de formes (transferts financiers, garde d'enfants...), dont l'une est le partage du logement entre générations successives aux âges adultes. Notre travail s'inscrit dans la lignée de ces études, puisqu'il porte d'une part sur les modes de cohabitation des jeunes adultes, et d'autre part sur ceux des personnes âgées. Cette analyse des modes de cohabitation repose sur la notion de ménage ${ }^{1}$. Cette dernière apparaît adaptée pour la production de statistiques puisqu'elle est utilisée " comme outil de collecte des données individuelles » (Lefranc, 1997: 53). Mais cette notion a aussi un sens d'un point de vue économique et social (Vincens, 1957), comme l'ont notamment souligné Van Imhoff et collab. : « Dans un large éventail de comportements humains en général, le ménage plutôt que l'individu est l'unité pertinente dans la prise de décisions " (Van Imhoff, Kuijsten, Hooimeijer et Van Missen, 1995: 1 [notre traduction]).

À l'instar de l'étude menée par Holdsworth (2000) qui compare la décohabitation en Espagne et en Grande-Bretagne en considérant que ces pays reflètent ce que l'on observe dans les pays du sud et du nord de l'Europe, notre analyse porte sur deux pays voisins, à savoir la France et l'Espagne. On considère que la France, pays d'Europe septentrionale, et l'Espagne, qui appartient à l'Europe méridionale, sont respectivement représentatifs des pays du nord et du sud de l'Europe. Par exemple, la décohabitation tardive des jeunes en Espagne est parfaitement représentative de ce que l'on observe dans le groupe des pays du sud de l'Europe (Cavalli et Galland, 1995; Reher, 1998). De plus, dans les pays du sud, notamment en Espagne et au Portugal, « la cohabitation intergénérationnelle est une pratique plus fréquente, et l'aide familiale aux personnes âgées dépendantes est fournie dans le cadre de la cohabitation des enfants " (Weber, 2011: 23). En outre, si plusieurs travaux ont souligné d'importantes différences infranationales - au niveau provincial espagnol concernant la décohabitation des jeunes notamment (Holdsworth, 1998; Holdsworth, Voast et Tranmer, 2002); au niveau départemental français concernant la proportion de ménages d'une seule personne (Ogden et Hall, 2004); sur la taille des ménages dans dix grandes villes françaises

1. La définition du ménage résulte de la combinaison (différente selon les années et selon les pays) de trois critères principaux : la cohabitation dans un même logement, un lien de parenté et l'existence de dépenses communes. 
(Ogden et Hall, 2000); au niveau individuel, communal et provincial à propos des modes de cohabitation des jeunes en Espagne (Vitali, 2009) les études sur les modes de cohabitations menées à un niveau régional demeurent rares en raison du manque de données le permettant.

Cette recherche ${ }^{2}$ a précisément pour but de vérifier, dans un premier temps, s'il existe une hétérogénéité spatiale des formes familiales entre les régions françaises et espagnoles, en analysant les modes de cohabitation des jeunes adultes âgés de 20 à 29 ans et des personnes âgées de plus de 65 ans. Cette étude sera menée à partir des échantillons de microdonnées de recensement fournis par IECM. Il s'agira, dans un second temps, de montrer que les résultats issus de cette analyse régionale masquent des disparités internes, à partir de l'exemple des cantons de la région Aquitaine et des données de recensement françaises. Nous retenons cette région car, comme on le verra, ce territoire présente des formes de cohabitation singulières, résultant de facteurs historiques et sociaux.

\section{DONNÉES ET CHOIX MÉTHODOLOGIQUES}

\section{Données utilisées}

L'obstacle principal auquel sont confrontés les chercheurs est la complexité dans l'obtention de données permettant d'effectuer des comparaisons internationales, notamment concernant la composition des ménages. Si les données de recensement permettent de réaliser des études à un échelon territorial fin (infranational et infrarégional), on assiste depuis quelques années à une remise en cause du recensement « classique » en Europe, les raisons principalement invoquées étant sa lourdeur et son coût. Cette remise en cause se traduit par un allègement des recensements dans la plupart des pays européens, avec la réduction du nombre de questions dans le questionnaire ou un espacement des passages (Begeot et Eggerickx, 1993; Valente, 2010). Il en découle un certain appauvrissement et parfois un problème de comparabilité dans le temps et dans l'espace, comme le soulignent Eggericks et Begeot : " L'harmonisation du contenu des recensements est bien évidemment la première des conditions afin d'assurer la

2. Cette recherche est menée dans le cadre du projet ANR Census, qui consiste à évaluer la couverture et la qualité de la base de données IECM (Integrated European Census Microdata) par des mesures démographiques. Trois équipes de recherche travaillent conjointement sur l'évaluation de cette base, dans le cadre du projet Census : l'Unité de comparaisons internationales de l'INED, l'Observatoire démographique européen et l'Institut d'études démographiques de l'Université de Bordeaux 4. 
comparabilité des résultats entre les pays » (Begeot et Eggerickx, 1993 : 1718). Jusqu'à présent, seule la mise en place d'enquêtes ponctuelles permettait de surmonter cet obstacle. Citons par exemple :

- l'enquête OASIS 3 , qui avait pour objectif de « déterminer les liens entre les variables personnelles, sanitaires et familiales, et diverses dimensions de la solidarité familiale, dans une perspective comparative transnationale » (Lowenstein, Katz et Mehlhaussen-Hassoen, 2003 : 52), a permis de mettre en exergue une solidarité familiale très forte dans les cinq pays couverts par l'étude (Norvège, Angleterre, Allemagne, Espagne et Israël) à l'égard des plus de 75 ans, mais avec des formes variables d'un pays à l'autre. Selon Lowenstein et collab. (2003: 69), «Les similarités et les différences observées entre ces pays pourraient traduire des disparités au niveau des normes culturelles/familiales, des comportements et des traditions de la politique sociale »;

- le projet FELICIE (Future Elderly Living Conditions in Europe Project) mené à partir de données d'enquêtes nationales et de l'ECHP (European Community Household $\mathrm{Panel}^{4}$ ) a permis de montrer que les caractéristiques sociodémographiques jouent le même rôle sur la probabilité d'appartenir à l'un des quatre types de ménage dans lesquels vivent les personnes âgées en Europe, "mis à part l'influence du fait d'être sans enfant et du genre, mais l'hétérogénéité géographique des types de ménage reste partiellement inexpliquée en raison de problèmes de comparabilité des sources de données en Europe » (Gaymu et collab. 2006 : 242);

- l'enquête SHARE5 (Survey on Health, Ageing and Retirement in Europe) sur la santé, le vieillissement et la retraite en Europe constitue un

3. L'enquête «Old Age and Autonomy: The Role of Service Systems and Intergenerational Solidarity » a été menée entre 2000 et 2003 dans 5 pays : Norvège, Angleterre, Allemagne, Espagne et Israël, dans le cadre d'un échantillon urbain représentatif composé de 1200 personnes (800 âgées de 25 à 74 ans et 400 de 75 ans et plus), dans chacun des cinq pays participants, soit au total 6 ooo personnes interrogées à l'aide d'un instrument d'enquête standardisé.

4. L'ECHP est une enquête annuelle menée entre 1994 et 2001 sur la base d'un questionnaire standardisé couvrant un large éventail de sujets : revenus, santé, éducation, logement, emploi... sur un échantillon d'environ 60500 ménages, soit à peu près 130 ooo personnes âgées de 16 ans et plus, dans 12 pays membres de l'Union européenne à l'origine, puis 15 ensuite (Belgique, Danemark, Allemagne, Espagne, Grèce, France, Irlande, Italie, Luxembourg, Pays-Bas, Autriche, Portugal, Finlande, Suède et Royaume-Uni).

5. Depuis 2004, l'enquête «Survey of Health, Ageing and Retirement in Europe » interroge à travers toute l'Europe un échantillon de ménages dont au moins un membre est âgé de 50 ans et plus. Ces ménages sont réinterrogés tous les deux ans en panel. La 
panel européen axé sur les questions sanitaires et socio-économiques liées au vieillissement. Elle a permis de dégager des résultats concernant la santé des séniors (les Européens du Nord sont plus riches et en meilleure santé, mais on vit plus longtemps dans le sud, Espagne, Grèce, Israël et Italie), la famille et les réseaux sociaux (la disparition de la famille est un mythe, au Nord les parents donnent plus tandis qu'au Sud ils reçoivent davantage...) ou la fin de la vie active des séniors (la spécificité des systèmes nationaux de protection sociale est à l'origine de différences de comportements);

- le programme GGP (The Gender and Generation Programme), complémentaire de l'enquête SHARE, qui prend en considération tous les âges adultes en insistant sur les relations intergénérationnelles. Ces enquêtes nationales et les bases de données contextuelles ont pour principal objectif «de fournir des données qui peuvent contribuer à une meilleure compréhension de l'évolution démographique et sociale et des facteurs qui influent sur ces développements, avec une attention particulière accordée aux relations entre parents et enfants (générations) et ceux entre les partenaires (des sexes) ${ }^{6}$. Le programme GGP met en évidence la diversification des parcours d'entrée dans l'âge adulte, les relations entre les générations, la question du soutien entre générations...

Les travaux réalisés à partir de toutes ces données d'enquêtes, qui portent sur la corésidence de différentes générations successives d'adultes en Europe, s'accordent notamment sur l'opposition entre les pays du sud et ceux du nord de l'Europe. Mais, par définition, ces enquêtes qui ne portent que sur un échantillon de la population nationale ne permettent pas, contrairement aux données de recensement, d'effectuer d'analyse à un échelon infranational. C'est pour pallier ces difficultés que le Minnesota Population Center et le centre d'études démographiques de Barcelone ont collaboré pour mettre en place, grâce à la participation de 18 instituts nationaux de statistiques, la base d'échantillons de microdonnées de recensement IECM (Integrated European Census Microdata). Cette base européenne intégrée contient des échantillons de microdonnées anonymisées d'environ 50 recensements. L'objectif est de fournir des données aux chercheurs afin de faciliter les comparaisons européennes dans le temps et dans l'espace. Nous avons exploité les données de la base IECM pour étudier la prévalence de la corésidence intergénérationnelle des adultes.

vague 4 (2010-2011), actuellement sur le terrain, est réalisée dans 20 pays européens. http :/www.irdes.fr/EspaceRecherche/Enquetes/SHARE/index.html

6. http://www.unece.org/the-population-unit/areas-of-work/generations-and-gender/ generations-and-gender/about-ggp.html 
La base de données IECM constitue le volet européen de la base IPUMS. IPUMS-International est un projet qui vise à collecter, harmoniser et diffuser des échantillons de microdonnées de recensement anonymisées de tous les pays du monde afin de les rendre utilisables par les chercheurs. L'objectif d'IPUMS est de permettre d'effectuer des comparaisons entre les pays à partir de microdonnées, grâce à des variables harmonisées « integrated ( (qui permettent la comparaison entre les pays), tout en disposant d'informations explicites sur les métadonnées et d'une documentation du recensement complète. Par ailleurs, IPUMS met également à disposition les données non harmonisées (variables brutes). Le principal avantage de la base de données IPUMS est que toutes les informations sont disponibles sur le site Internet et que l'échantillon est suffisamment grand pour obtenir un niveau de significativité élevé.

\section{La comparabilité des données et les limites}

Il est important de considérer les différences entre les deux pays (France et Espagne) concernant la méthodologie, les questionnaires, les définitions et les concepts utilisés dans les recensements, car certaines différences peuvent avoir une influence considérable sur l'analyse, en particulier dans une perspective comparative (Begeot et Eggerickx, 1993; Lefranc, 1997). Voici un aperçu des principales questions qu'il convient de se poser afin de vérifier la comparabilité des données.

\section{La question de la définition du ménage}

Les critères utilisés pour la définition du ménage sont strictement identiques dans le recensement français de 1999 et dans le recensement espagnol de 2001. Ils font référence à la notion de ménage-logement, c'est-à-dire que le ménage est formé de l'ensemble des occupants d’un même logement.

\section{La question de la définition des membres du ménage (de jure ou de facto)}

La définition utilisée pour identifier les membres du ménage diffère entre la France et l'Espagne pour ces deux recensements. Ainsi, la population recensée correspond à la population :

- de facto en Espagne en 2001 : toute personne est recensée à l'endroit où elle se trouve au moment du recensement;

- de jure en France en 1999 : les personnes recensées sont celles vivant habituellement dans le ménage, même si elles sont absentes pour une durée plus ou moins longue au moment de l'enquête. 
Cette divergence dans la définition des membres du ménage introduit une discordance concernant la prise en compte de certaines catégories, comme les étudiants pensionnaires. L'utilisation de la définition de jure en France implique que les étudiants sont plutôt recensés dans le ménage de leur famille, c'est-à-dire qu'ils sont réintégrés dans le ménage de leurs parents. Tandis qu'en Espagne, avec l'utilisation de la définition de facto, les étudiants sont davantage recensés sur le lieu de leurs études. Par conséquent, on peut aboutir à une surreprésentation des jeunes en corésidence familiale résultant de la définition de jure en France, et une proportion plus faible de cette corésidence des jeunes selon la définition de facto en Espagne.

Cette discordance dans les définitions utilisées peut également avoir un impact sur la proportion de personnes âgées recensées en institution, en particulier ceux qui ne résident pas à plein temps en maison de retraite. Ainsi, en France, on peut obtenir une sous-estimation du nombre des plus de 65 ans en institution, car suivant la définition de jure ils peuvent être recensés dans le ménage privé auquel ils appartiennent et non en « collectif », tandis qu'en Espagne ils sont recensés dans l'institution.

\section{La question de la désignation de la personne de référence du ménage}

La personne de référence du ménage est désignée selon une procédure automatique dans les deux cas.

En Espagne en 2001, est considérée comme personne de référence la personne énumérée en premier lieu dans la feuille de recensement, le premier répondant dans le questionnaire. Cette définition de la personne de référence est donc a priori totalement arbitraire. Néanmoins, les questionnaires remis par l'Institut national de statistiques espagnol lors du recensement de 2001 étaient personnalisés, c'est-à-dire pré-remplis à partir des informations issues du padrón municipal de façon à faire apparaître en premier dans chaque feuille de ménage « la personne la plus adéquate ${ }^{8}$ » (INE, 2001).

Toutefois, la méthode de détermination de l'ordre d'apparition demeurant obscure, il est très difficile de jauger la qualité de ce procédé.

7. Le padrón municipal est un registre administratif de population où figurent les résidents de chaque commune.

8. Définition de la personne de référence énoncée en page 44 du projet de recensement 2001 de l'Institut national de statistiques espagnol (INE). Disponible en ligne (PDF) : http ://www.ine.es/censo2001/proceno1.pdf 
En France, depuis 1982, le chef de ménage est devenu «personne de référence du ménage ». Il est désigné par une procédure automatique qui assigne la personne de référence selon plusieurs critères : le sexe, l'âge et l'activité. La règle sur laquelle repose ce processus clairement spécifiée par l'INSEE :

Cette procédure automatique $[\ldots]$ ne prend en compte que les trois personnes les plus âgées du ménage (classées par ordre décroissant) et considère leurs relations potentielles :

— si le ménage compte une seule personne, cette dernière est la personne de référence;

— si le ménage compte deux personnes : si elles sont de sexe différent et identifiées comme formant un couple, l'homme est la personne de référence; sinon la personne de référence est la personne active la plus âgée, ou si aucune des deux n'est active, la personne la plus âgée;

— si le ménage compte trois personnes ou plus : si un couple formé d'un homme et d'une femme est identifié, l'homme du couple est la personne de référence; sinon la personne de référence est la personne active la plus âgée, ou si aucune des trois personnes considérées n'est active, la personne la plus âgée. (INSEE, 2008: 5).

D'aucuns se sont interrogés sur la pertinence de cette détermination arbitraire de la personne de référence en France (Courson, 1982), qui peut aboutir à une vision partiale de la société, notamment avec la désignation systématique de l'homme du couple comme personne de référence. Ce favoritisme vis-à-vis du genre masculin est en décalage par rapport à la place de la femme dans la société (De Saint Pol, Denay et Monso, 2004), alors que «la répartition du pouvoir au sein du ménage est devenue plus démocratique, $[\ldots]$ les revenus du ménage proviennent souvent du travail des deux conjoints » (Wall, 1996 : 93).

\section{Les choix méthodologiques}

L'objectif est d'analyser les modes de cohabitation des jeunes adultes âgés de 20 à 29 ans et des personnes âgées de 65 ans ou plus, dans les régions françaises et espagnoles, à partir des données de la base IECM. En utilisant ces données pour effectuer des comparaisons entre les régions françaises et espagnoles, on cherche à déceler d'éventuels effets frontière dans les modes 
de cohabitation, c'est-à-dire que l'on cherche à savoir si cette répartition est homogène dans toutes les régions françaises d'une part et dans toutes les régions espagnoles d'autre part, ou si au contraire il existe des disparités régionales dans les régions du sud de la France ou du nord de l'Espagne vis-à-vis du reste de leur pays respectif, tout en vérifiant si des spécificités nationales sont observables à partir des échantillons de microdonnées de recensement. Autrement dit, on cherche à déceler l'éventuelle influence d'une ou plusieurs aires culturelles communes (basques et catalanes essentiellement) situées à cheval sur la frontière franco-espagnole.

Dans cette étude, on utilise les échantillons du recensement français de 1999 et du recensement espagnol de 2001, échantillons au 1/20 dans les deux cas, représentatifs des logements au niveau national. Les données de la base IECM sont disponibles au niveau "NUTS2 ", qui correspond au niveau administratif des régions françaises et à celui des communautés autonomes espagnoles. Cependant, les résultats concernant les îles Canaries ne sont pas présentés dans les cartes et on exclut de l'analyse les villes espagnoles de Ceuta et Melilla, ainsi que les DOM-TOM français, en raison de contextes culturels, géographiques et économiques qui diffèrent de la métropole.

On s'intéresse d'une part aux modes de cohabitation des jeunes adultes âgés de 20 à 29 ans qui quittent de plus en plus tardivement le foyer parental (Singly, 2000; Galland, 2000; Rougerie et Courtois, 1997; Fernandez Cordon, 1997), et d'autre part à ceux des personnes âgées de plus de 65 ans susceptibles d'être en début de dépendance (Gaymu et collab. 2007; Gaymu, Festy, Poulain et Beets, 2008).

Les catégories de ménages peuvent être définies en fonction de plusieurs critères : selon le lien de parenté entre les membres du ménage, selon le nombre de générations cohabitantes, selon l'état matrimonial des individus... L'éventail des typologies possibles est large, allant de la classification des ménages mise au point par Peter Laslett — qui différencie les solitaires, les ménages sans famille, les ménages simples, les familles élargies et les ménages multiples - (Laslett et Wall, 1972: 28-34) à des typologies extrêmement complexes pouvant atteindre 88 catégories comme ce fut le cas dans une étude norvégienne (As, 1986). Pour mener à bien cette étude, étant données les variables disponibles dans les données françaises et espagnoles et eu égard de nos sujets d'étude, nous avons construit une typologie des ménages selon le mode de cohabitation ${ }^{9}$, en utilisant entre

9. Cette typologie des modes de cohabitation s'inspire la classification établie par Fokkema et Liefbroer à partir des informations contenues dans l'enquête « Labor 
autres la variable du lien de parenté avec la personne de référence (voir tableau 1). On différencie ainsi la vie en solitaire, la vie en couple seul, la corésidence familiale, la vie en institution et la cohabitation avec des membres non apparentés. La corésidence familiale peut être subdivisée en trois sous-modes de corésidence familiale : les couples avec enfants, les familles monoparentales et la cohabitation multigénérationnelle.

TABLEAU 1 Typologie des ménages selon le mode de cohabitation

\begin{tabular}{|c|c|}
\hline Type de ménage & Caractéristiques \\
\hline Personne seule & Ménage composé d'un seul et unique membre \\
\hline Couple seul & $\begin{array}{l}\text { Ménage composé du chef de ménage et de son époux/se ou } \\
\text { partenaire, sans enfants }\end{array}$ \\
\hline \multicolumn{2}{|l|}{ Corésidence familiale } \\
\hline - soit famille nucléaire & Ménage composé d’un couple avec enfant(s) \\
\hline $\begin{array}{l}\text { - soit famille } \\
\text { monoparentale }\end{array}$ & Ménage composé d’un unique parent avec enfant(s) \\
\hline $\begin{array}{l}\text { - soit cohabitation } \\
\text { multigénérationnelle }\end{array}$ & Ménage composé de plus de deux générations \\
\hline Non apparentés & $\begin{array}{l}\text { Ménage comprenant un individu non apparenté aux autres } \\
\text { membres du ménage }\end{array}$ \\
\hline En ménage collectif & Personne qui vit dans un établissement collectif \\
\hline
\end{tabular}

Cette typologie nous permet d'étudier la répartition des personnes âgées selon le mode de cohabitation en isolant les ménages collectifs, c'està-dire les personnes placées en institution.

En revanche, on fait le choix de ne tenir compte que des ménages privés pour mener une analyse des modes de cohabitation des jeunes adultes $^{10}$. Par conséquent, en excluant la catégorie « en ménage collectif », la typologie des ménages utilisée sera légèrement différente de celle exposée ci-dessus en ce qui concerne les jeunes adultes, sans la catégorie " collectif ». On dispose donc des catégories suivantes :

Force Survey » : « living with parent(s) », « living in a one-person household (living alone) », « living as a couple», « living as a couple with child(ren)», « living as alone parent » et « other» (Fokkema et Liefbroer, 2008).

10. En France, la proportion de jeunes adultes résidant en «collectif » varie de 3 à $5 \%$ selon la région, tandis qu'en Espagne, cette part varie de 0,2 à $0,8 \%$ dans les communautés autonomes de l'Espagne métropolitaine. Par conséquent, en décidant d'exclure les « collectifs » de l'analyse, on n'écarte qu'une fraction négligeable des jeunes adultes. 
- personne seule,

- en couple seul,

- en corésidence familiale (soit famille nucléaire, soit famille monoparentale, soit cohabitation multigénérationnelle),

- non apparentés.

Cependant, s'agissant des jeunes adultes âgés de 20 à 29 ans, il convient de faire une distinction supplémentaire en ce qui concerne la corésidence familiale, selon que le jeune adulte réside encore avec ses parents, ou selon qu'il a décohabité et a créé une nouvelle famille. On précisera donc si le jeune adulte est " en corésidence familiale — cohabitant avec les parents », ou « en corésidence familiale — ayant créé une nouvelle famille».

\section{RÉSULTATS}

Analyse régionale de la cohabitation des jeunes adultes âgés de 20 à 29 ans $^{11}$, à partir des échantillons de recensement

On observant les cartes présentant la distribution des modes de cohabitation des jeunes adultes, on distingue de fortes spécificités nationales. En France, les jeunes semblent vivre de façon plus indépendante - en couple ou en solitaire - qu'en Espagne, où les jeunes vivent plus souvent en corésidence familiale ou avec des membres non apparentés. La corésidence familiale des jeunes adultes est relativement forte dans les deux pays (plus de $50 \%$ ) mais elle est nettement supérieure en Espagne (cartes 1 à 4). Ces divergences entre la France et l'Espagne peuvent refléter l'opposition en termes de normes de responsabilité familiales souvent signalées entre pays du sud et du nord de l'Europe : les Européens du Sud sont non seulement plus attachés à l'institution familiale que les Européens du Nord, mais adhèrent également plus fortement qu'eux aux normes d'obligation familiale (Kalmijn et Saraceno, 2008; Daatland et Herlofson, 2003).

Au-delà de cette opposition entre ces deux pays, on remarque quelques particularités infranationales. En effet, la corésidence familiale est plus forte dans le nord de la France et en Corse, et plus faible dans le sud et l'ouest de l'hexagone. Aussi, cette corésidence est moindre dans les communautés autonomes du nord-est de l'Espagne.

11. On a choisi de ne pas distinguer les 20-24 ans des 25-29 ans afin de ne pas alourdir la lecture des résultats. En outre, ce choix se justifie car les disparités régionales ici présentées varient peu selon le groupe d'âge. 
CARTES 1 À 4 Proportion (en \%) des jeunes adultes de 20-29 ans selon le mode de cohabitation, dans les régions françaises et espagnoles

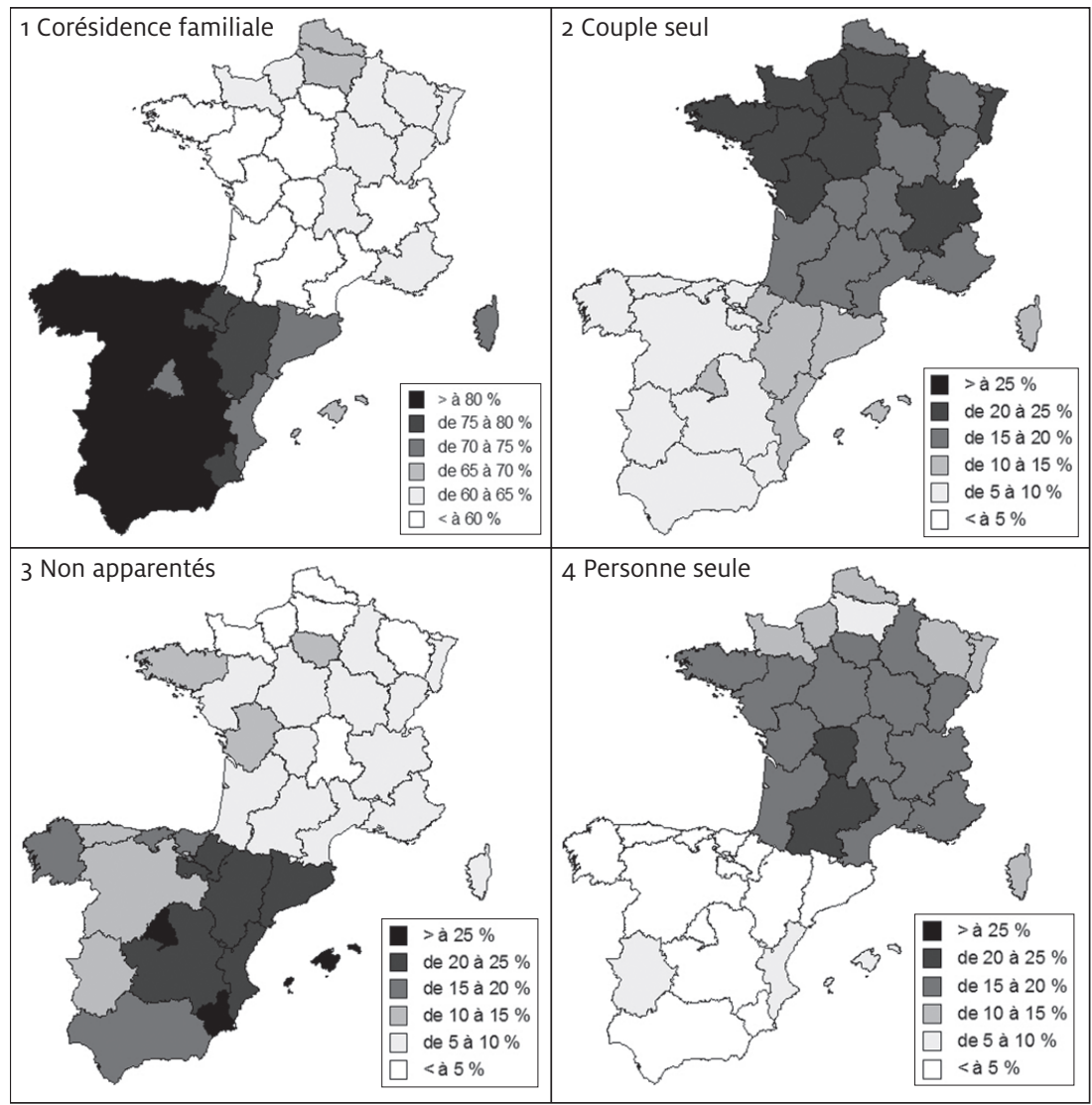

Il convient de distinguer les jeunes qui résident toujours avec leurs parents, c'est-à-dire qui n'ont pas encore décohabité, et ceux qui ont créé une nouvelle famille. Il apparaît ainsi une opposition radicale entre les deux pays : les jeunes ne vivent quasiment pas de façon indépendante en Espagne, puisqu'ils cohabitent plus massivement avec leurs parents, tandis que ce phénomène est plus rare en France, où les jeunes adultes ont davantage décohabité et créé une nouvelle famille (cartes 5 et 6). Ces résultats confirment une décohabitation plus tardive des jeunes en Espagne (Holdsworth et collab. 2002; Ayllón, 2009; Vitali, 2010), contrairement à ce que l'on observe dans les pays du nord de l'Europe tels que la France. Les raisons avancées pour expliquer ce retard à la décohabitation sont multiples. Certains évoquent l'allongement des études en Espagne, qui se 
traduit par un délai de plus en plus long avant l'insertion professionnelle, et donc un allongement de la cohabitation avec les parents (Oinonen, 2008). D'aucuns soutiennent que cette décohabitation tardive résulte de l'absence d'opportunités économiques pour les jeunes dans les pays d'Europe du Sud (Ghidoni, 2002; Fernandez Cordon, 1997), qui se traduit par une hausse du chômage des jeunes conduisant à une dépendance économique prolongée envers le foyer parental (Lesthaeghe et Moors, 2000). D'ailleurs, Wallace a montré que les jeunes sans emploi ont deux fois plus de chances de vivre avec leurs parents que les jeunes qui travaillent (Wallace, 1987). En outre, contrairement à la position « interventionniste» des pays du nord de l'Europe, les traditions du sud de l'Europe - plus «familialistes" - conduiraient plusieurs générations à cohabiter sous le même toit (Galland et Lemel, 2007).

CARTES 5 ET 6 Proportion (en \%) des jeunes adultes de 20-29 ans en corésidence familiale, dans les régions françaises et espagnoles

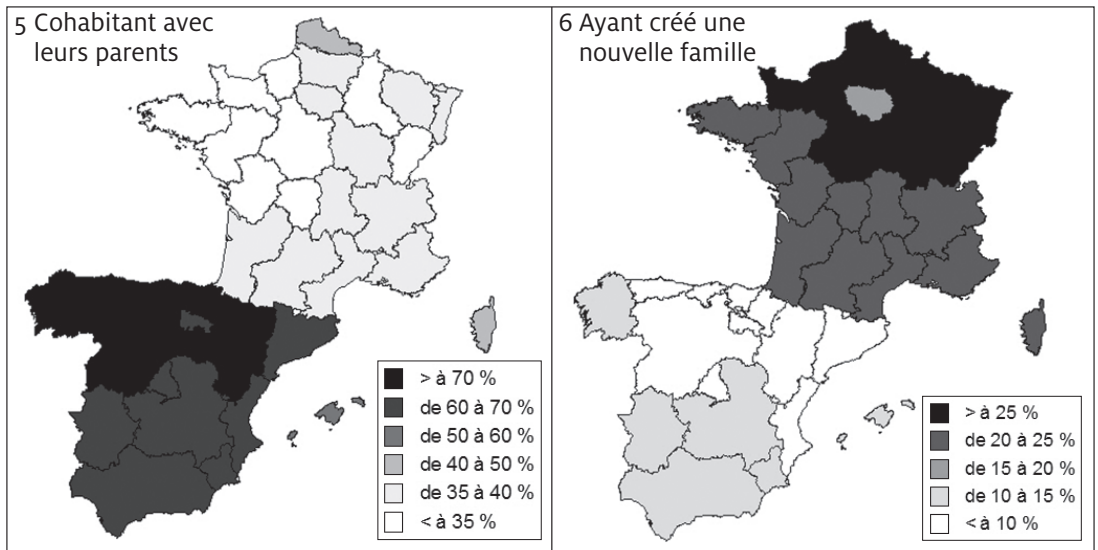

La corésidence familiale des jeunes adultes masque des modalités différentes selon les régions (cartes 7 à 11). Ainsi, si l'on affine notre typologie de la corésidence familiale, on constate que :

- la cohabitation multigénérationnelle, de trois générations ou plus, est plus forte en Espagne - pays familialiste (Lowenstein et collab. 2003) - , mais demeure rare en France. Ce mode de cohabitation correspond quasi-exclusivement à des jeunes qui habitent toujours avec leurs parents (entre $1 \%$ et moins de $4 \%$ des jeunes en cohabitation multigénérationelle, selon la région, ont créé une nouvelle famille). 
- la cohabitation en famille monoparentale est supérieure en Espagne et en Provence-Côte-d'Azur-Corse (PACA-Corse). Il s'agit quasi exclusivement de jeunes adultes qui habitent encore avec l'un de leurs parents. Moins de $3 \%$ des jeunes adultes âgés de 20 à 29 ans habitent de façon indépendante en famille monoparentale avec leur enfant(s) en ayant décohabité, que ce soit dans les régions françaises ou espagnoles.

- la cohabitation sous forme de famille nucléaire est particulièrement importante en Espagne, tandis qu'en France elle concerne moins de jeunes dans le sud que dans le nord. Mais si l'on distingue les jeunes en couple avec enfant(s) qui habitent encore avec leurs parents de ceux qui ont créé une nouvelle famille, on découvre que l'Espagne se caractérise par des jeunes adultes qui cohabitent toujours avec leurs parents, tandis qu'en France les jeunes ont plus souvent décohabité, surtout dans les régions du nord (cartes 10 et 11).

\section{L'analyse régionale de la cohabitation des personnes âgées de plus de 65 ans à partir des échantillons de recensement}

La répartition des personnes de plus de 65 ans selon le mode de cohabitation diffère entre la France et l'Espagne, mais aussi au sein de chaque pays (cartes 12 à 16). La corésidence familiale des personnes âgées est beaucoup plus forte en Espagne, notamment dans les communautés autonomes du nord et en Andalousie. En France, la proportion des plus de 65 ans cohabitant avec leur famille est nettement plus faible qu'en Espagne, mais on constate que la corésidence est plus fréquente dans les régions frontalières et en Corse. Ainsi, on découvre une continuité au-delà de la frontière franco-espagnole en matière de corésidence familiale des personnes âgées.

Cette continuité peut être le reflet d'aires culturelles communes (basques et catalanes) situées à cheval sur la frontière franco-espagnole. Or, selon Emmanuel Todd : "l'Europe [...] est sur le plan familial un conservatoire de formes archaïques» (Todd, 2011:15). Dans certaines zones du nord de l'Espagne, les normes de transmission patrimoniales étaient basées sur l'indivisibilité de l'héritage, se caractérisant par un système familial troncal (Reher, 1996). La répartition des systèmes familiaux traditionnels était la suivante :

- la famille nucléaire - ne comprenant qu'un couple avec ou sans enfants, et n'associant jamais plus de deux générations - triomphait en Espagne centrale et méridionale, 
CARTES 7 À 11 Proportion (en \%) des jeunes adultes de 20-29 ans selon le mode de corésidence familiale, dans les régions françaises et espagnoles

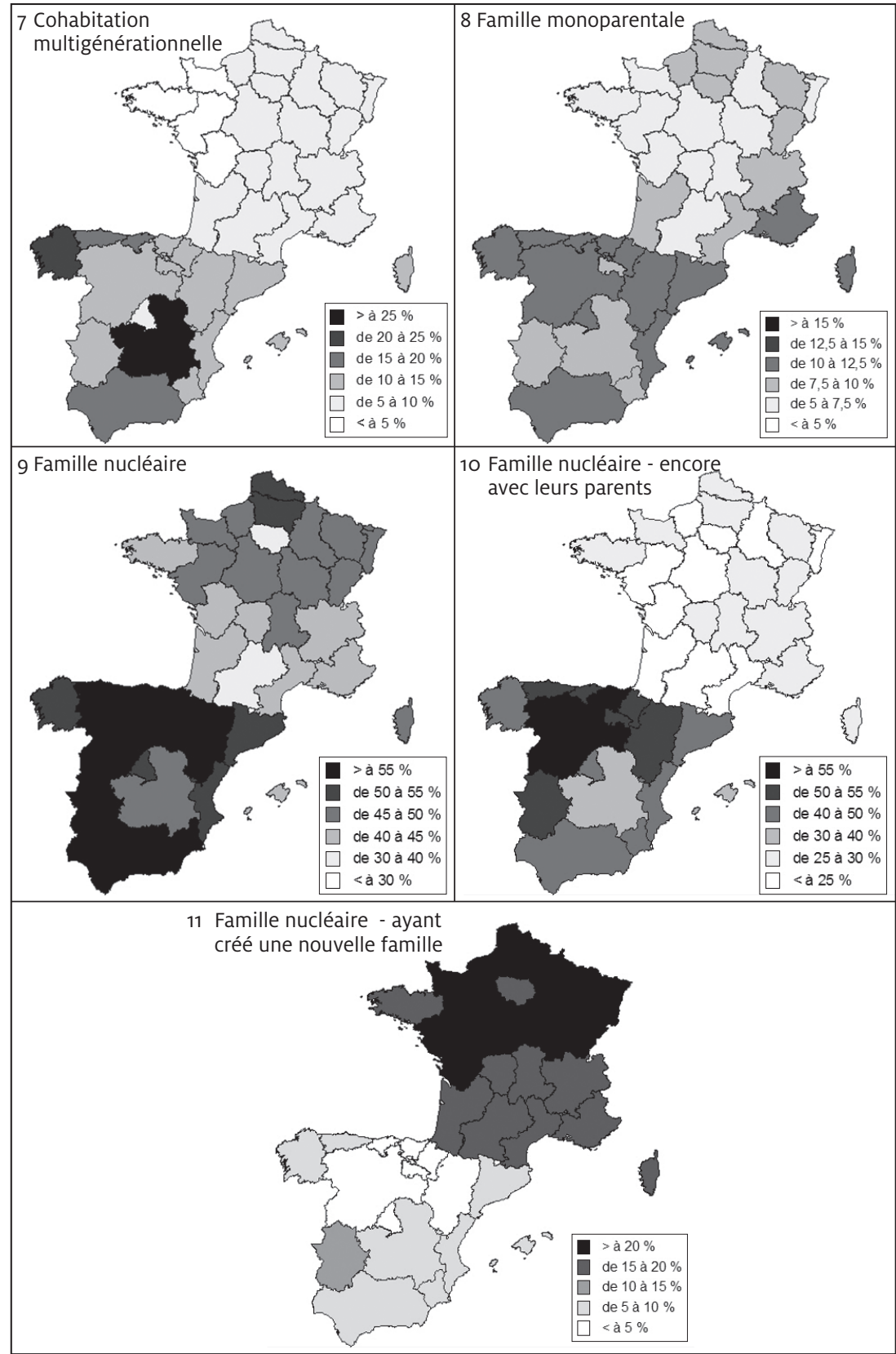


- la famille souche - le couple produit des enfants et désigne un successeur unique qui doit corésider avec ses parents, associant plus de deux générations lorsque ce dernier à des enfants - était prédominante dans le sud-ouest de la France et le nord de la péninsule ibérique (Todd, 2011).

Ainsi, on peut interpréter cette répartition des personnes âgées selon les modes de cohabitation dans les régions françaises et espagnoles comme une persistance de la famille souche dans les régions frontalières du sud de la France et du nord de l'Espagne.

En contrepartie, la part des personnes de plus de 65 ans qui vivent en institution - ici sous le vocable ménage collectif - est quasiment nulle en Espagne. La part des personnes de plus de 65 ans qui vivent seules est très faible en Espagne - notamment dans les communautés autonomes du nord - , autour de $15 \%$, contre $30 \%$ en France.

Ce contraste dans la prise en charge des personnes âgées en France et en Espagne découle également probablement de la divergence des systèmes de sécurité sociale. Certains auteurs l'on souligné, "l'Espagne demeure une société "familialiste" traditionnelle dotée d'un réseau de services formels moins développé pour les personnes âgées » (Lowenstein et collab., 2003 : 68-69). Le recours aux maisons de retraite est donc moins fréquent en Espagne.

Enfin, la proportion de personnes âgées vivant « en couple seul » est systématiquement plus élevée en France qu'en Espagne. Certaines communautés autonomes du nord de l'Espagne - Galice, Cantabrie et Navarre - présentent une proportion de personnes âgées vivant en « couple seul » particulièrement faible.

La part des personnes de plus de 65 ans qui cohabitent avec des «non apparentés » est quasiment nulle dans toutes les régions françaises et espagnoles.

Ainsi, la répartition des personnes de plus de 65 ans en Espagne est plutôt représentative de celle observée dans les pays du sud de l'Europe, avec une cohabitation plus forte avec les enfants, et une moindre vie en solitaire ou en institution.

De nombreux auteurs ont évoqué que, dans les pays d'Europe du Nord, les personnes âgées vivent plus souvent seules ou en institution, alors que la cohabitation multigénérationnelle est plus répandue dans les pays d'Europe du sud (Iacovou, 2002; Tomassini et collab., 2004; Gaymu et collab., 2008). Cette diversité des modes de cohabitation des personnes âgées peut refléter les différences des systèmes familiaux historiques, des 
CARTES 12 À 16 Proportion (en \%) des personnes de plus de 65 ans selon le mode de corésidence, dans les régions françaises et espagnoles

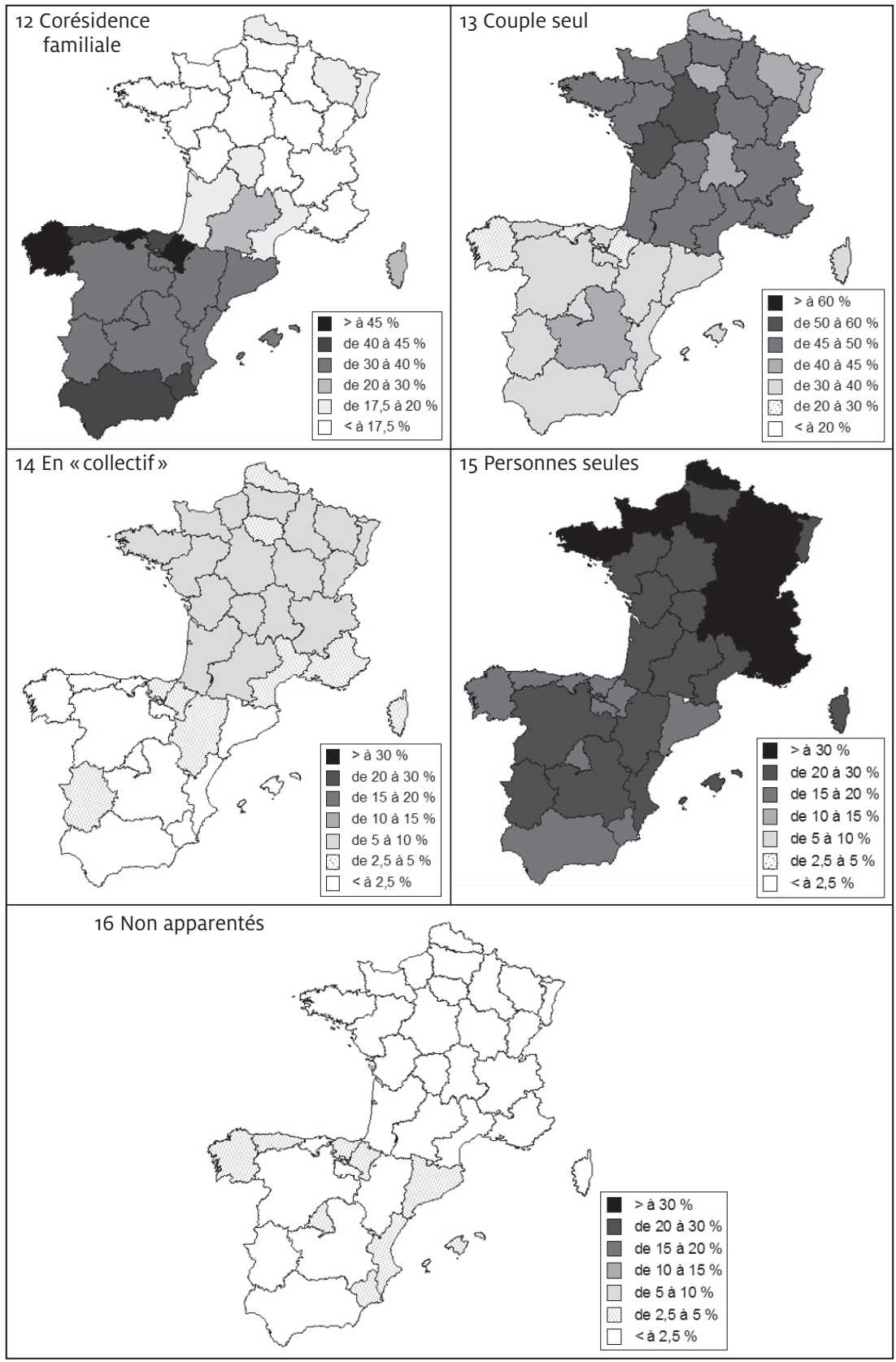


liens familiaux relativement faibles au nord de l'Europe mais forts dans les pays méditerranéens (Reher, 1998).

CARTES 17 À 19 Proportion (en \%) des personnes de plus de 65 ans selon le mode de corésidence familiale, régions françaises et espagnoles

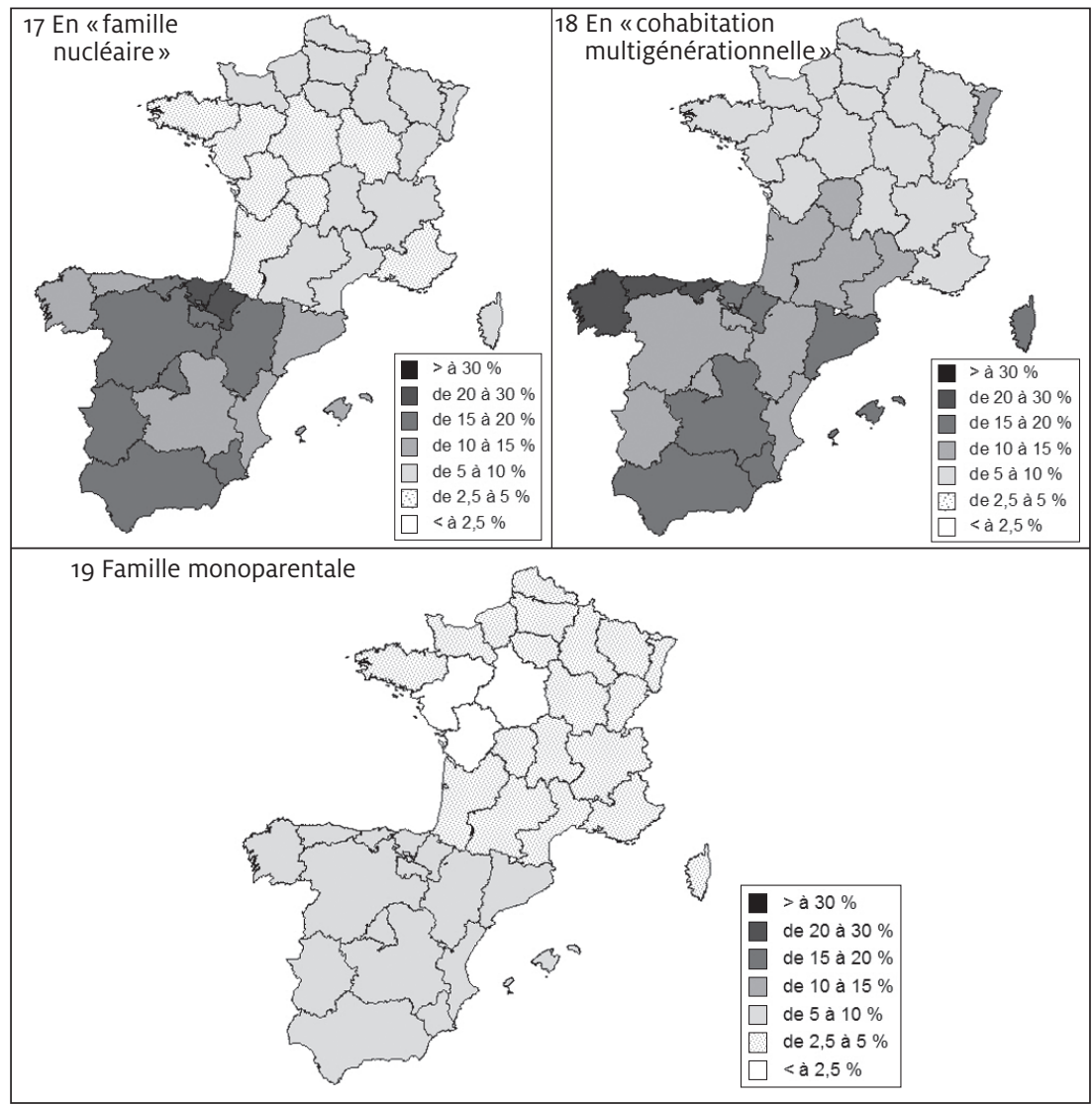

En effet, la corésidence des personnes âgées est plus forte en Espagne, et si l'on affine l'analyse de cette corésidence familiale (cartes 17 à 19), on découvre que :

- la proportion des plus de 65 ans qui habitent dans un ménage en «famille monoparentale » est très faible dans les régions françaises et espagnoles;

- la part des plus de 65 ans qui vivent en «famille nucléaire » est nettement supérieure en Espagne, notamment dans les communautés autonomes du Pays Basque et de Navarre; 
- la part des personnes de plus de 65 ans qui habitent en « cohabitation multigénérationnelle » est nettement supérieure en Espagne, $10 \%$ contre $5 \%$ en France. Dans les régions frontalières du sud de la France, la part des personnes de plus de 65 ans en « cohabitation multigénérationnelle » est plus proche de celle observée dans les communautés autonomes espagnoles que de celle observée dans les régions françaises voisines. Il semblerait donc qu'il existe une continuité au-delà de la frontière en matière de « cohabitation multigénérationnelle $»^{12}$.

Ainsi, il semblerait que l'on assiste à une persistance des « modèles familiaux traditionnels ». En réalité, si cette persistance des modèles traditionnels est validée concernant les personnes âgées (Gaymu et collab., 2008), ce n'est pas le cas en ce qui concerne les jeunes adultes. Les modèles de cohabitation traditionnels en corésidence familiale sont respectés par les personnes âgées, probablement en raison d'un effet de génération. Les personnes de plus de 65 ans appartiennent aux générations les plus anciennes, précisément celles ayant connu le plus ces traditions. Tandis que la cohabitation des jeunes est un phénomène nouveau.

La base d'échantillons de microdonnées de recensement IECM ne permet pas d'affiner davantage cette analyse, notamment à une échelle territoriale plus fine. Les données de la base IECM sont disponibles au niveau des provinces espagnoles, mais uniquement au niveau des régions pour la France. Or il existe une importante hétérogénéité à l'intérieur de certaines régions françaises. Nous allons illustrer cette hétérogénéité à partir de l'exemple des cantons dans la région Aquitaine. L'Aquitaine est la région située à l'extrême sud-ouest de la France, à la frontière avec l'Espagne. L'analyse des modes de cohabitation au niveau des cantons français n'est possible qu'à partir des données exhaustives de recensement, disponibles sur le site Internet de l'Institut National de la Statistique et des Études Économiques (INSEE) français ${ }^{13}$. Cela devrait nous permettre de vérifier les conclusions tirées de l'analyse à partir des échantillons de recen-

12. De même que pour les jeunes, on a choisi de ne pas distinguer les 65-74 ans des 75-84 ans et des 85 ans et plus, afin de ne pas alourdir la lecture des résultats. En outre, si la répartition des personnes âgées selon le mode de cohabitation varie considérablement selon le groupe d'âge, la proportion de personnes âgées en " cohabitation multigénérationnelle » est à tous les âges toujours plus élevée dans les communautés autonomes espagnoles et dans les régions du sud de la France que dans les régions françaises.

13. http ://www.insee.fr/fr/ 
sement, en particulier à propos des permanences anciennes dans les modes de cohabitation.

\section{L'analyse des différences spatiales dans les modes de cohabitation à partir des données exhaustives de recensement}

Les fichiers détaillés utilisés ici se rapportent au recensement français de 2006, alors que les échantillons de recensement utilisés précédemment concernent le recensement français de 1999 et le recensement espagnol de 2001. Néanmoins, on considère que la répartition des personnes âgées et des jeunes adultes n'a pas connu de bouleversements majeurs entre 1999 et 2006 en Aquitaine (Bergouignan, Dasre et Kersuzan, 2008). En l'espèce, on a utilisé le fichier de personnes relatif à la région Aquitaine avec des données localisées au niveau du canton ou de la ville du lieu de résidence de l'individu. Ce fichier fournit les caractéristiques sociodémographiques de chaque individu et celles de son ménage.

À partir de ces données exhaustives de recensement, et en utilisant la même typologie des ménages que précédemment (voir tableau 1), nous avons vérifié s'il existe une homogénéité dans la répartition des personnes âgées et des jeunes adultes selon le mode de cohabitation en Aquitaine, ou au contraire si l'on observe une hétérogénéité, non visible à partir des échantillons de recensement.

L'analyse de la cohabitation des jeunes adultes dans les cantons d'Aquitaine à partir des données exhaustives du recensement

La répartition des jeunes adultes âgés de 20 à 29 ans selon leur mode de cohabitation est hétérogène en Aquitaine (cartes 20 à 23). En effet, le pourcentage de chaque mode de cohabitation varie considérablement selon les cantons aquitains, avec une proportion pouvant aller du simple au quadruple d'un canton à l'autre pour un même mode.

Par exemple, si l'on s'intéresse plus particulièrement à la part des jeunes adultes en corésidence familiale, on découvre qu'elle est inférieure à $20 \%$ dans certains cantons, mais dépasse les $70 \%$ dans d'autres. En fait, la corésidence familiale des jeunes est plus faible dans les cantons ruraux et plus élevée dans les cantons urbains. Or les cantons urbains constituent les zones les plus habitées. L'image lissée de la région Aquitaine que l'on obtenait à partir des échantillons de recensement résultait donc d'une surreprésentation des cantons urbains les plus peuplés, ce qui cachait les fortes disparités infrarégionales. 
CARTES 20 À 25 Proportion (en \%) des jeunes adultes, selon le mode de cohabitation, cantons d'Aquitaine

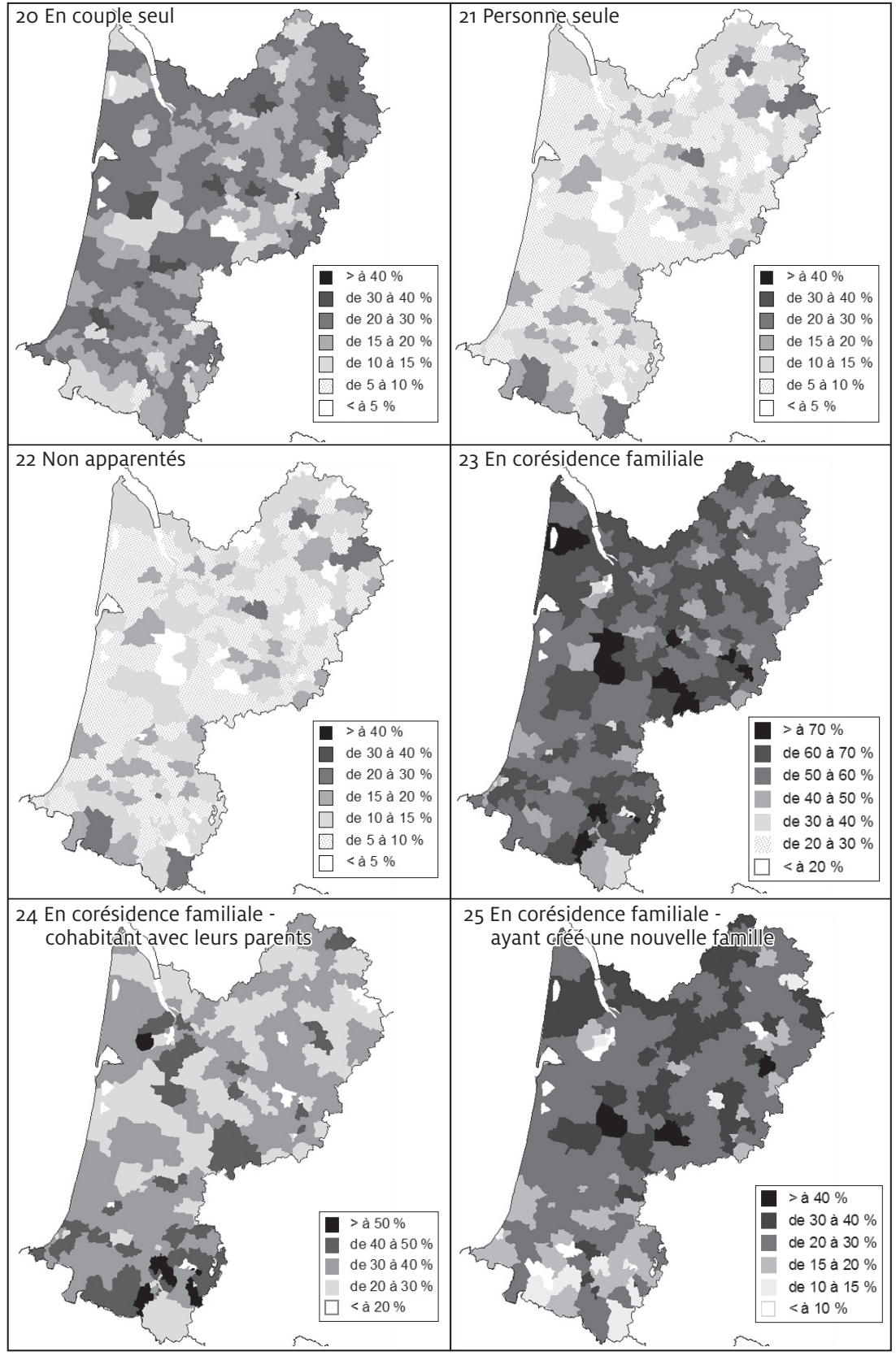


Les cantons du sud de l'Aquitaine se singularisent par une cohabitation majoritaire des jeunes adultes avec leurs parents (cartes 24 et 25).

L'analyse de la cohabitation des personnes âgées dans les cantons d'Aquitaine à partir des données exhaustives du recensement

L'analyse régionale de la répartition des personnes de plus de 65 ans effectuée à partir des échantillons de recensement nous a permis de mettre en évidence une continuité au-delà de la frontière en termes de corésidence familiale des personnes âgées. On a constaté que la proportion des plus de 65 ans qui corésident avec leur famille est beaucoup plus forte dans les communautés autonomes du nord de l'Espagne et dans les régions du sud de la France. En analysant cette répartition au niveau des cantons aquitains, on distingue une très forte hétérogénéité au sein de la région (cartes 26 à 30). Ainsi, il apparaît que la corésidence familiale est supérieure dans les cantons du sud de l'Aquitaine, notamment au Pays Basque. Or le Pays Basque se caractérise historiquement par un système traditionnel de familles souches, c'est-à-dire par la corésidence des personnes âgées avec leur(s) enfant(s) et associant plus de deux générations (Todd, 2011).

La vision cantonale permet de mettre en lumière des différences infrarégionales importantes en matière de corésidence familiale chez les jeunes adultes âgés de 20 à 29 ans et chez les plus de 65 ans. Néanmoins, elles n'ont pas la même nature. La corésidence des jeunes constitue un phénomène nouveau, peu hétérogène, tandis que chez les plus de 65 ans, la corésidence tient davantage de la tradition, notamment d'une permanence du modèle historique des familles souches dans l'extrême sud-ouest de l'Aquitaine. 
CARTES 26 À 30 Proportion (en \%) des personnes de plus de 65 ans, selon le mode de cohabitation, cantons d'Aquitaine

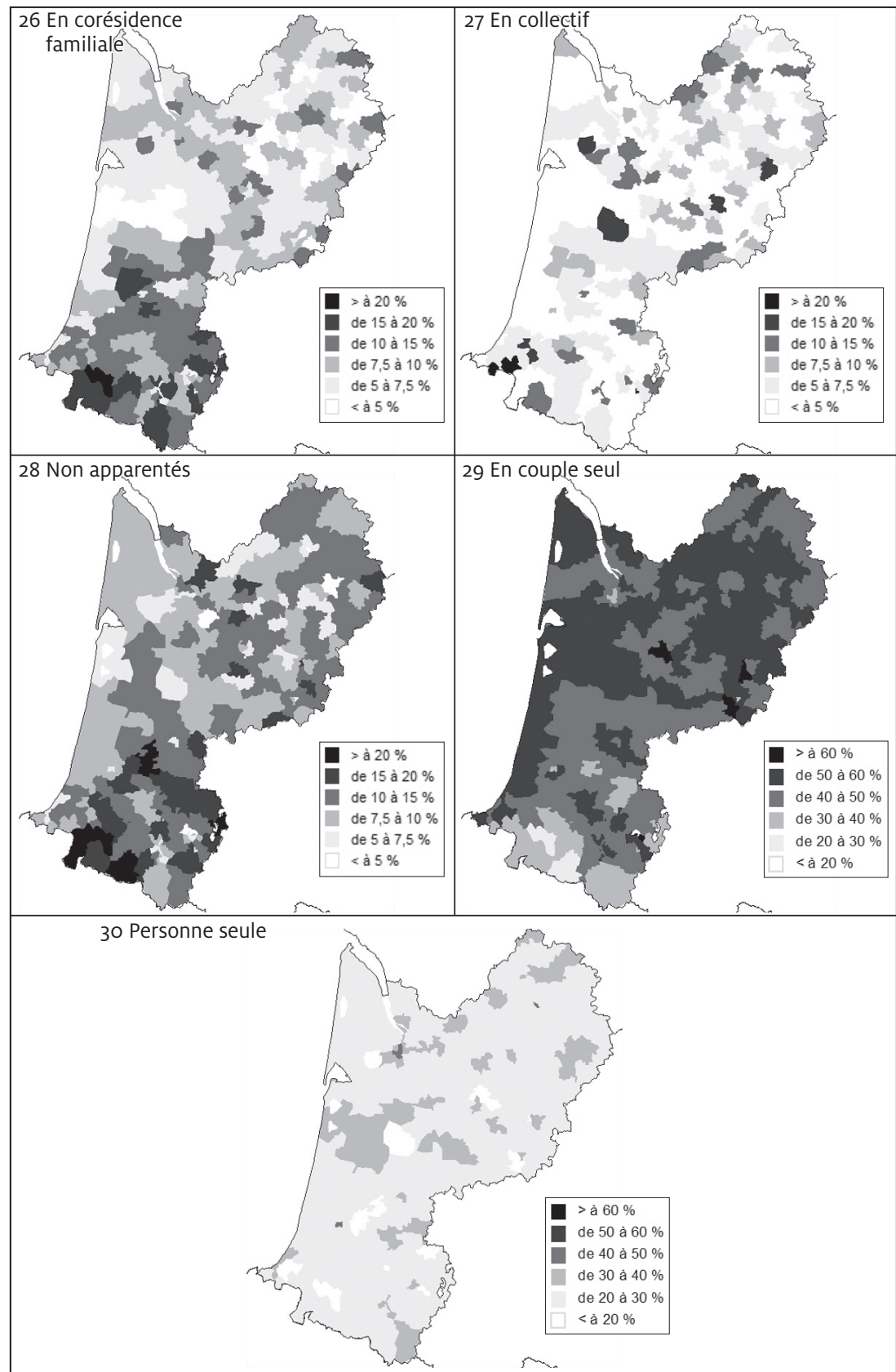




\section{CONCLUSION}

Ce travail a permis de mettre en évidence, à partir des échantillons de recensement de la base IECM, une hétérogénéité spatiale des formes familiales, même si, comme on l'a évoqué, quelques limites de comparabilité persistent.

Si les données IECM nous ont permis de mener une analyse régionale comparative en France et en Espagne, elles ne permettent pas de mener une analyse à un échelon territorial très fin. En outre, le manque d'homogénéité dans les définitions utilisées par les deux pays dans leur recensement constitue une difficulté supplémentaire. En pratique, le manque de continuité dans le temps et dans l'espace de la notion de ménage pose problème, et peut empêcher les comparaisons spatiales et temporelles. Par exemple, le ménage revêt deux conceptions différentes dans les pays européens : d'aucuns appréhendent le ménage à partir du logement commun, on parle de ménage-logement - Danemark, France, Espagne -, tandis que d'autres pays se réfèrent au ménage-budget, sur la base d'une unité de budget. Non seulement la définition n'est pas la même selon les pays, mais elle peut également varier au cours des recensements. Ainsi, la définition du ménage utilisée dans le recensement espagnol de 2001 est basée uniquement sur le critère de la cohabitation, sans tenir compte de la condition de " partager des dépenses communes ", à la différence du recensement précédent en 1991. Ce changement de définition rend la comparaison difficile, car il a une influence sur le nombre de ménages et sur leur taille. En effet, dans les pays où le ménage correspond au ménage-logement, le nombre de ménages est sensiblement moins élevé qu'ailleurs (Kaufmann, 1994).

Cette étude a non seulement permis de déceler une opposition entre la France et l'Espagne, mais aussi des disparités infranationales. La frontière géographique entre la France et l'Espagne est nettement plus marquée en ce qui concerne la fréquence de la cohabitation familiale des jeunes adultes que s'agissant de celle des plus de 65 ans. Cette divergence entre la France et l'Espagne concernant les modes de cohabitation des jeunes de 20 à 29 ans confirme le contraste entre le nord et le sud de l'Europe évoqué par de nombreux auteurs, qui se traduit par une décohabitation plus tardive dans les pays du sud.

Par ailleurs, on distingue des disparités infranationales plus fortes chez les personnes âgées corésidantes que chez les jeunes adultes. On a mis en évidence une continuité patente au-delà de la frontière en matière de corésidence familiale des personnes âgées, qui résulte d'une permanence des modèles familiaux traditionnels dans le nord de l'Espagne et dans les 
régions du sud de la France. Les disparités concernant la fréquence de la cohabitation des jeunes adultes sont moins fortes, et correspondent à un phénomène nouveau, peu hétérogène et détaché des "modèles familiaux traditionnels ». En confrontant ces résultats à ceux obtenus à partir des données cantonales du recensement français de 2006, on peut déceler une hétérogénéité supplémentaire des modes de cohabitation au sein de l'Aquitaine, région frontalière. La vision cantonale permet de mettre en lumière des différences infrarégionales importantes en matière de corésidence familiale, mais il n'en demeure pas moins que ces disparités sont moins fortes chez les jeunes que chez les personnes âgées. On discerne clairement une concentration particulière du modèle traditionnel de la "famille souche » dans l'extrême sud-ouest de l'Aquitaine. Cette hétérogénéité est dissimulée par les échantillons de recensement IECM au niveau régional, mais peut être révélée grâce aux données exhaustives de recensement fournies par l'INSEE au niveau des cantons.

Il serait intéressant de prolonger ces travaux en élargissant le cadre de cette recherche à d'autres pays européens, en particulier en raison du contexte de crise économique dans lequel l'Europe se trouve actuellement. Cette crise économique engendre des politiques d'austérité qui touchent pour le moment en priorité les pays du sud de l'Europe. Nous verrons à l'avenir si le modèle traditionnel familial déjà observable dans le sud de l'Europe va s'amplifier pour renforcer les solidarités familiales, et donc la cohabitation. En effet, cette crise tend aujourd'hui à s'étendre aux pays du nord de l'Europe, réputés plus individualistes. Si ces pays du nord connaissaient des politiques aussi austères que celles appliquées dans le sud de l'Europe, ce qui risquerait d'ébranler leurs systèmes sociaux publics, ces populations pourraient être conduites à favoriser davantage les solidarités intergénérationnelles, plus à l'image de ce que l'on peut observer dans les pays du sud. Dès lors, la différence entre pays interventionnistes et pays familialistes pourrait s'estomper et la solidarité familiale se substituer aux systèmes de sécurité sociale.

\section{BIBLIOGRAPHIE}

Algava, E. 2002. "Les familles monoparentales en 1999 ", Population, 57, $4-5: 733-758$.

As, D. 1986. Shared Households of Adults who do not live with Spouse but who do not live Alone. Oslo, Norges Byggforskninstitutt.

Attias-Donfut, C. (dir.). 1995. Les solidarités entre les générations. Vieillesse, familles, État. Paris, Nathan, $352 \mathrm{p}$. 
Ayllón, S. 2009. "Poverty and living arrangements among youth in Spain, 19802005 ", Demographic Research, 20, 17 : 403-434.

Begeot, F. et T. Eggerickx. 1993. "Les recensements en Europe dans les années 1990. De la diversité des pratiques nationales à la comparabilité internationale des résultats ", Population, 48, $6: 1705-1732$.

Bergouignan, C., A. Dasre et C. Kersuzan. 2008. La population girondine : des spécificités globales aux logiques territoriales. Rapport de recherche remis au Conseil Général de la Gironde, 60 p.

BLöss, T. 2005. «Relations entre générations et inégalités sociales », Informations sociales, $5,125: 72-79$.

Bongants, J. 2006. "How Long Will We Live?», Population and Development Review, $32: 605-628$.

Bonvalet, C. et J. Ogg. 2009. Les baby-boomers : une génération mobile. Paris, Éditions de l'Aube, $252 \mathrm{p}$.

Bozon, M. et C. Villeneuve-Gokalp. 1995. «L'art et la manière de quitter ses parents ", Population et sociétés, 297.

Cavalli, A. et Galland, O. 1995. Youth in Europe. Social Change in Western Europe. London : Pinter, $160 \mathrm{p}$.

Courson, J.-P. 1982. "Les ménages n'auront plus de chef », Économie et statistiques, $149: 47-55$.

Daatland, S. O. et K. Herlofson. 2003. «Les normes de responsabilité familiale dans les pays européens : contrastes et similarités ", Retraite et société, 38 , $1: 15-47$.

De Saint Pol, T., A. Denay et O. Monso. 2004. " Ménage et chef de ménage: deux notions bien ancrées », Travail, genre et sociétés, La Découverte, 11 : 63-78.

De Santis, G. 2003. "Les aspects démographiques d'un système équitable de transferts intergénérationnels ", Population, 58, 6: 667-706.

Desplanques, G. et M. Saboulin. 1986. «Mariage et premier enfant : un lien qui se défait ", Économie et Statistique, 187, 1:31-45.

Fernandez Cordon, J.A. 1997. «Youth Residential Independence and Autonomy. À Comparative Study ", Journal of Family Issues, 18, 6 : 576-607.

Festy, P. et L. Prokofieva. 2006. "La pauvreté et l'évolution des structures familiales en Russie depuis 1990 ", Revue d'études comparatives Est-Ouest, 37, $2: 109-134$.

Fleury, C., M. Borsenberger., S. Cassilde et N. Lorentz. 2011, Solidarités intergénérationnelles. Le cas de la génération pivot, Working Paper, Differdange, CEPS/INSTEAD, 2011-015, $104 \mathrm{p}$.

Fokkema, T. et A. C. Liefbroer. 2008. « Trends in living arrangements in Europe : Convergence or divergence? », Demographic Research, 19, 36 : 1351-1418.

Galland, O. 2000. «Entrer dans la vie adulte: des étapes toujours plus tardives mais resserrées ", Économie et statistique, 337-338: 13-36.

Galland, O. 2004. Sociologie de la jeunesse, Paris, Colin, 247 p. 
Galland, O. et Y. Lemel. 2007. Valeurs et cultures en Europe. Paris, Éditions La Découverte, $120 \mathrm{p}$.

Gaymu, J., C. Delbes, S. Springer, A. Binet, A. Desesquelles, S. Kalogirou et U. ZIEGLER. 2006. "Determinants of the living arrangements of older people in Europe ", European Journal of Population, 22, $3:$ 241-262.

Gaymu, J., P. Ekamper et G. Beets. 2007. «Qui prendra en charge les Européens âgés dépendants en 2030? ", Population, 62, $4: 789-822$.

Gaymu, J., P. Festy, M. Poulain et G. Beets. 2008. Future Elderly Living Conditions in Europe. Paris, Ined, 320 p.

Ghidoni, M. 2002. Determinants of young Europeans' decision to leave the parental household. London, Royal Economic Society Annual Conference 2002, no 85 .

Haskey, J. 1993. "Formation and dissolution of unions in different countries of Europe ", dans A. Blum et J. L. Rallu (dir.), European Population. Vol. 2 : Demographic Dynamics, London : 211-229.

Holdsworth, C. 1998. "Leaving Home in Spain : A Regional Analysis », International Journal of Population Geography, 4:341-360

Holdsworth, C. 200o. «Leaving Home Britain and Spain », European Sociological Review, 16, 2 : 201-222.

Holdsworth, C. et M. Irazoqui Solda. 2002. « First Housing Moves in Spain : An Analysis of Leaving Home and First Housing Acquisition », European Journal of Population, $18:$ :-19

Holdsworth, C., D. Voast et M. Tranmer. 2002. «Leaving Home in Spain : When, Where and Why? ", Regional Studies, 36, 9: 989-1004

IACOvou, M. 2002. "Sharing and Caring : older Europeans' living arrangements », Journal of Applied Social Science Studies, 122 : 1-32.

INE. 2001. Censos de poblacion y viviendas 2001-Proyecto. Madrid, $125 \mathrm{p}$.

INSEE. 2008. Fichiers détail : documentation. INDCVIZC : Fichier Individus (localisation au canton-ou-ville). $26 \mathrm{p}$.

Kalmijn, M. et C. SARACENO. 2008. «À comparative perspective on intergenerational support : Responsiveness to parental needs in individualistic and familialistic countries », European Societies, 10, 3: 479-508.

Kaufmann, J.-C. 1994. «Les ménages d'une personne en Europe », Population, 49, 4-5: 935-958.

Kiernan, K. 2002. "The State of European Unions: An Analysis of FFS Data on Partnership Formation and Dissolution », dans M. Macura et G. C. N. Beets. (dir.), Dynamics of Fertility and Partnership in Europe : Insights and Lessons From Comparative Research, Volume 1, New York/Geneva, United Nations : 57-76.

Kohler, H. P., F. C. Billari et J. A. Ortega. 2002. "The Emergence of Lowestlow Fertility in Europe During the 1990s ", Population and Development Review, $28: 641-680$.

Laslett, P. et R. Wall. 1972. Household and Family in Past Time. Cambridge University Press, $623 \mathrm{p}$. 
Lefranc, Ch. 1997. "Des difficultés et de l'intérêt de la statistique des ménages ", Statéco, 87-88: 53-63.

Lesthaeghe, R. 1991. The second demographic transition in Western countries : an interpretation. IPD Working paper, 1991-2. Brussels, Centrum Sociologie, Vrije Universiteit.

Lesthaeghe, R. 1995. "The Second Demographic Transition in Western Countries : An Interpretation », dans K. O. Mason et A. M. Jensen (dir.), Gender and Family Change in Industrialized Countries, Oxford, Clarendon Press : 17-62.

Lesthaeghe, R. et G. Moors. 2000. « Recent Trends in Fertility and household Formation in the Industrialized World ", Review of Population and Social Policy, $9: 121-170$.

Lowenstein, A., R. Katz et D. Mehlhaussen-Hassoen. 2003. "Une comparaison transnationale de la solidarité intergénérationnelle », Retraite et société, $38: 49-75$.

Millar, J. et A. Warman. 1996. Family Obligations in Europe. London : Family Policy Studies Centre.

Ogden, P. E. et R. Hall. 200o. " Households, reurbanisation and the rise of living alone in the principal French cities, 1975-1990 », Urban Studies, 37, 2 : 367-390.

Ogden, P. E. et R. Hall. 2004. "The second demographic transition, new household forms and the urban population of France during the 1990s ", Transactions of the Institute of British Geographers, 29, $1: 88-105$.

Oinonen, E. 2008. Families in Converging Europe : A Comparison of Forms, Structures and Ideals. New York: Palgrave Macmillan, $212 \mathrm{p}$.

Prioux, F. 2003. "L'âge à la première union en France: Une évolution en deux temps », Population, 58, 4-5: 623-644.

ReHer, D.-S. 1996. La familia en espana, pasado y presente. Madrid, Alianza editorial.

Reher, D.-S. 1998. " Family ties in Western Europe : persistent contrasts », Population and development review, $2: 203-234$.

Rougerie, C. et J. Courtors. 1997. «Une étape du passage à l'âge adulte : l'emploi qui compte ", Population, 52, $6: 1297-1327$.

Singly, F. 2000. "Penser autrement la jeunesse », Lien social et politiques, 43: 9-15. Sовотка, T. 2004. Postponement of Childbearing and Low Fertility in Europe, Amsterdam, Dutch University Press, 298 p.

Todd, E. 2011. L'origine des systèmes familiaux. Tome I : L'Eurasie. Gallimard, 756 p. Tomassini, C., K. Glaser, D.A. Wolf, M. Van Groenou et E. Grundy. 2004. «Living arrangements among older people : an overview of trends in Europe and the USA ». Population trends, $115: 24-34$.

Valente, P. 2010. «Comment la population est-elle recensée dans les pays européens en 2010?", Population \& Sociétés, 467.

Van De KaA, D.J. 1987. «Europe's Second Demographic Transition », Population Bulletin, 42, $1: 1-57$. 
VAn De Velde, C. 2007. «La dépendance familiale des jeunes adultes en France. Traitement politique et enjeux normatifs », dans S. PAugam (dir.), Repenser la solidarité : l'apport des sciences sociales, Paris, PUF : 315-333.

Van Himhoff, E., A. Kuijsten, P. Hooimeijer et L. Van Wissen. 1995. «Introduction ", dans E.Van Himhoff, A. Kuijsten, P. Hooimeijer et L. Van Wissen, Household demography and household modelling, New-York, Plenum Press : 1-15.

Vincens, J. 1957. "La notion de ménage et son utilisation économique », Revue économique, 8 , $3: 412-438$.

Vitali, A. 2009. The impact of regional factors on leaving home in Spain : A multilevel approach. Milan, Università Bocconi, Carlo F. Dondena Centre for Research on Social Dynamics (DONDENA), working paper $n^{0} 17,19 \mathrm{p}$.

Vitali, A. 2010. «Regional differences in young Spaniard's living arrangement decisions : A multilevel approach », Advances in life course research, 15: 97-108.

WALL, R. 1996. "Comparer ménages et familles au niveau européen : problèmes et perspectives ", Population, 51, 1: 93-115.

WallaCE, C. 1987. For richer, for poorer : growing up in and out of work. London, Tavistock.

WEBER, F. 2007. «Qu'est-ce que la protection rapprochée? Réciprocité, solidarité quotidienne et affiliation symbolique », dans S. PAUGam (dir.), Repenser la solidarité. L'apport des sciences sociales, Paris, PUF : 187-204.

Weber, F. 2011. Handicap et dépendance. Drames humains, enjeux politiques. Paris, Éditions Rue d'Ulm, 76 p.

\section{ABSTRACT}

\section{Béatrice VALDES}

The heterogeneity of patterns of cohabitation of those aged between 20 and 29 and over 65 in French and Spanish regions, based on census data

This study of the patterns of cohabitation of young adults aged between 20 and 29 and of people aged over 65 , from census samples of the IECM database, highlights the spatial heterogeneity of patterns of cohabitation, showing not only an opposition between France and Spain, but also subnational disparities between French and Spanish regions. In terms of family co-residence of older people, we discover a continuity across the national boundary, resulting from the persistence of traditional family models in the regions of northern Spain and of southern France. Disparities in the prevalence of cohabitation among young adults are less strong, and correspond to a new phenomenon which is relatively homogeneous and detached from "traditional family models." At the cantonal level, data obtained from the cantonal French census data of 2006 highlight important sub-regional differences in terms of family co-residence. Here we can clearly identify a particular concentration of the traditional model of "root family" in the extreme southwest of Aquitaine. 